\title{
Skin hydration in postmenopausal women: argan oil benefit with oral and/or topical use
}

\author{
Kenza Qiraouani Boucetta ${ }^{1}$, Zoubida Charrouf ${ }^{2}$, Abdelfattah Derouiche ${ }^{3}$, Younes Rahalii ${ }^{1}$, Yahya Bensouda ${ }^{1}$ \\ ${ }^{1}$ Research Team on Formulation and Biopharmacy, Research Center for Drug, Faculty of Medicine and Pharmacy, \\ University Mohammed V-Souissi, Rabat, Morocco \\ ${ }^{2}$ Faculty of Sciences, University Mohammed V-Agdal, Rabat, Morocco \\ ${ }^{3}$ Faculty of Sciences, Ben Msik Hassan II University, Casablanca, Morocco
}

\begin{abstract}
The aim of this study was to evaluate the effect of daily consumption and/or application of argan oil on skin hydration in postmenopausal women.

Material and methods: Sixty postmenopausal women consumed butter during the stabilization period and were randomly divided into two groups for the intervention period: the treatment group absorbed alimentary argan oil $(n=30)$ and the control group olive oil $(n=30)$. Both groups applied cosmetic argan oil in the left volar forearm during a sixty days' period. Evaluation of skin hydration, i.e. transepidermal water loss (TEWL) and water content of the epidermis (WCE) on both volar forearms of the two groups, were performed during three visits at D0, D30 and after sixty days (D60) of oils treatment.

Results: The consumption of argan oil has led to a significant decrease in TEWL $(p=0.023)$ and a significant increase in WCE $(p=0.001)$. The application of argan oil has led to a significant decrease in TEWL $(p=0.01)$ and a significant increase in WCE $(p<0.001)$.

Conclusions: Our findings suggest that the daily consumption and application of argan oil have improved the skin hydration by restoring the barrier function and maintaining the water-holding capacity.

Key words: postmenopausal women, argan oil, skin hydration.
\end{abstract}

\section{Introduction}

Lipids play a key role in the structure of the skin, giving it control of the vital role of hydration and protection against dehydration. This protection is provided by the outermost layer of the epidermis, stratum corneum, consisting of flattened dead cells rich in keratin (corneocytes), capable of trapping water molecules using the natural moisturizing factors [1]. Corneocytes, are embedded in a lipid-rich intercellular matrix, consisting essentially of cholesterol, ceramides and free fatty acids [2]. Most of them are derived from the lipid content of lamellar bodies, formed within the keratinocytes of stratum granulosum in the presence of appropriate enzymes, during the process of keratinization. These lipids will be subsequently organized into lamellar bilayers in order to ensure the barrier function [3] giving the skin a waterproof aspect for keeping water in skin layers.

The disruption of the barrier function of the skin results in an increase in transepidermal water loss (TEWL) [4], and consequently a decrease in water content of the epidermis (WCE), which constitute the two main characteristics of dry skin [5].
During menopause, the decrease in endogenous estrogen levels affects negatively the homeostasis of the estrogen target organs including the skin, which becomes more predisposed to develop dryness [6]. This physiological fact led us to choose the population of postmenopausal women as the test population for this work.

Hormone replacement therapy (HRT) has been used to reduce these disturbances of the skin during menopause [6]. A beneficial effect on the hydration of the skin has been shown, but many pathophysiological events were triggered in the long-term use [7]. Except HRT, most of currently known emollients are only used externally to remedy this skin problem.

The purpose of this study was to evaluate the effect of daily consumption and topical application of an exogen natural lipid, argan oil, rich in linoleic acid (essential fatty acid), on skin hydration in postmenopausal women, as measured by the skin barrier function and water-holding capacity using non-invasive biophysical methods. 


\section{Material and methods}

\section{Material}

One hundred postmenopausal women were enrolled in our study; 60 of them met the following criteria. Inclusion criteria: menopause confirmed by dosages of estradiol (E2) and follicle-stimulating hormone (FSH) $(\mathrm{E} 2<30 \mathrm{pg} / \mathrm{ml}$ and $\mathrm{FSH}>40 \mathrm{IU} / \mathrm{l})$ and absence of skin diseases. Exclusion criteria: use of HRT, use of moisturizing products and dietary supplements.

The sixty postmenopausal women selected have given their informed consent and the study was approved by the Ethical Committee for Biomedical Research of the Faculty of Medicine and Pharmacy, University Mohammed V Souissi, Rabat, Morocco (Fig. 1).

\section{Products}

- Butter

- Moroccan olive oil

- Alimentary and cosmetic argan oil (provided by TARGANTE with ECOCERT label)
Argan oil has been widely used for millennia in the diet of the southwestern Moroccans. Today, its consumption has proved a positive effect in prevention of certain diseases such as hypercholesterolemia [8], heart disease [9], diabetes [10], etc. which have a greater incidence in postmenopausal women [11]. Previously, the use of cosmetic argan oil was limited in traditional medicine to the treatment of several skin problems [12] among the Berbers.

Spontaneously, cosmetic argan oil has become a staple in the preparation of moisturizers [13]. Scientific confirmation of this moisturizing effect would comfort and validate this instinctive tendency.

\section{Study protocol (Fig. 2)}

In this open-label trial, there were two diet periods. In diet period I (baseline diet), all the participants consumed $25 \mathrm{~g}$ /day of the same brand of butter for 2 weeks to stabilize the lipid profile [14] backed up by its pre- and post-intervention analysis, taken by consortium colleagues who realized the lipid profile monitor-

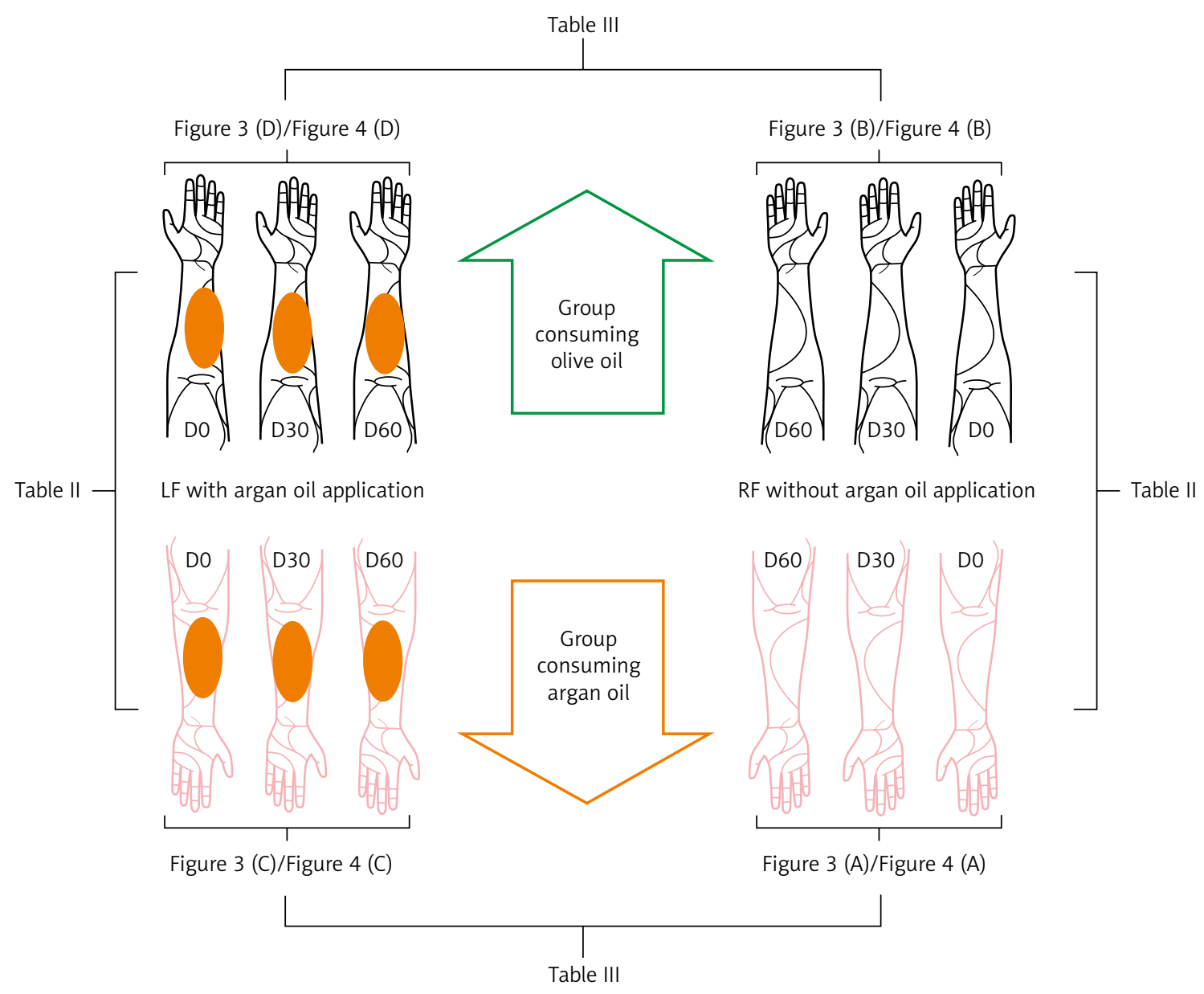

Fig. 1. Recapitulative scheme of the statistical results of transepidermal water loss (TEWL) and water content of epidermis (WCE) during the phase of intervention 
The phase of recruitment and stabilization of volunteers postmenopausal women (PMW)
The phase of intervention

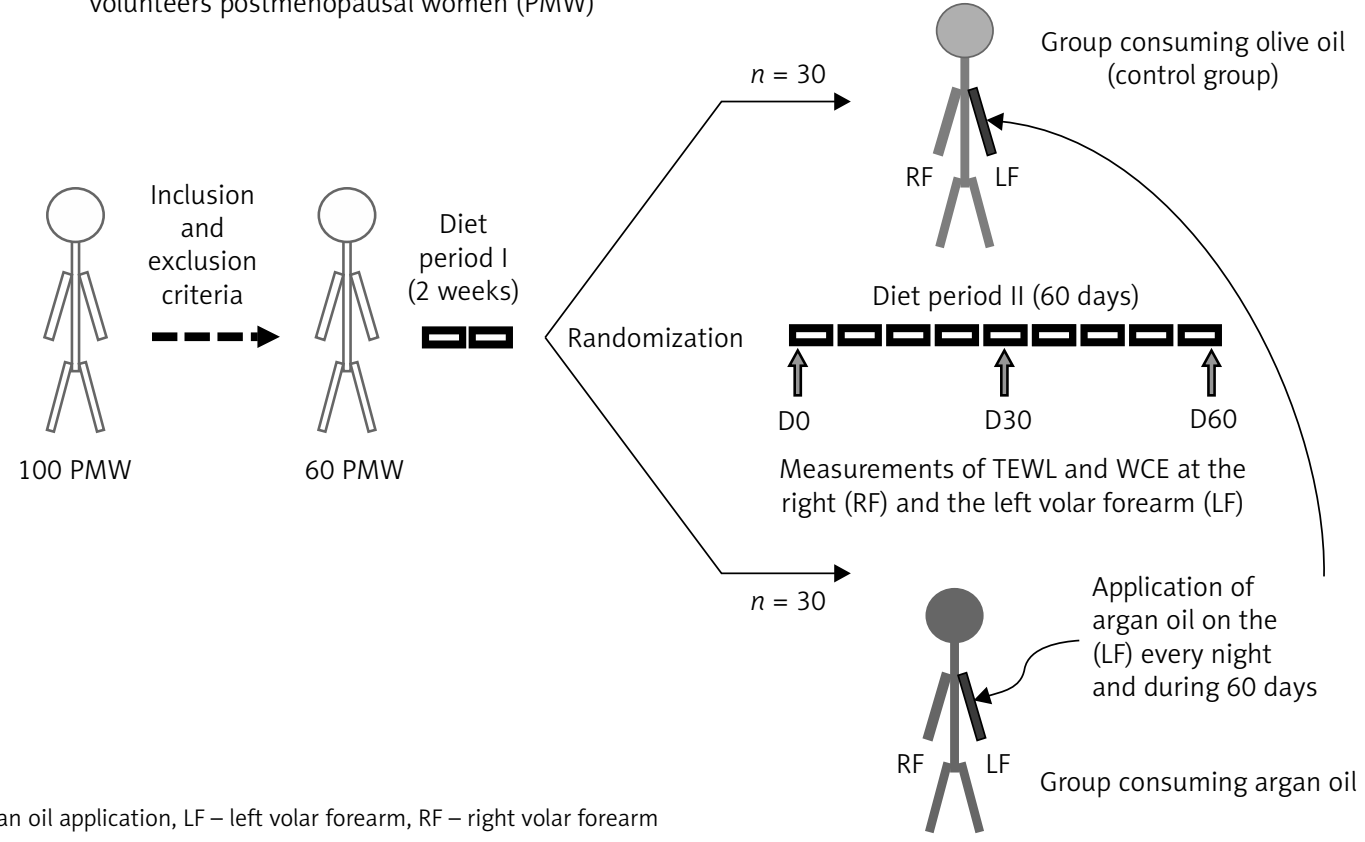

Fig. 2. Overall protocol of the study

ing. In diet period II, the 60 participants were randomly assigned to the two following diet groups using the "Data - Select cases - Random sample of cases" procedure in SPSS (Version10, SPSS Inc., Chicago, IL): one group of 30 participants, consumed $25 \mathrm{ml} /$ day of argan oil and the second group of 30 participants consumed $25 \mathrm{ml} /$ day of olive oil as controls during a sixty days' period. In parallel, the two groups were asked to apply every night about $240 \mathrm{mg}\left(2 \mathrm{mg} / \mathrm{cm}^{2}\right)$ corresponding to 10 drops of cosmetic argan oil on the left volar forearm (LF). During the study, the participants were forbidden to use any other skin care products on their volar forearms and were asked to adjust their eating habits to the average composition of the traditional Moroccan table food (or food basket) in order to limit the effect of the diet fat content which can affect the participants differently.

Cosmetic argan oil, and alimentary argan and olive oil bottles were delivered to participants every thirty days by the research staff, in order to meet the participants and ensure their adherence to the study protocol. The residual alimentary and cosmetic oil bottles were collected at the time of the next delivery in order to check the obedience of participants to treatment allocated. Olive oil was chosen as a control in order to increase patient compliance in the control group because it is widely known for its status as an affordable and healthy oil.

In the two groups, evaluation of skin hydration, i.e., TEWL and WCE at the right volar forearm (RF) and the LF, were performed in the morning, during three visits: at D0 (before starting oil consumption and application),
D30 (after thirty days of oil consumption and application) and D60 (after sixty days of oil consumption and application).

At each visit, all the participants stabilized during fifteen minutes in order to adjust to the climate-controlled room conditions (temperature $22 \pm 1^{\circ} \mathrm{C}$, relative humidity $56 \pm 1 \%$ ) before measurements.

\section{Bioengineering methods}

The measurements of TEWL and WCE were performed with a Tewameter TM300 and a Corneome$\operatorname{ter}^{\oplus} 825$, respectively, which were mounted on a Multi Probe Adapter ${ }^{\circledast}$ MPA5 (Courage and Khazaka Electronic $\mathrm{GmbH})$.

The measuring principle of the TEWL is based on the Fick's diffusion law indicating the quantity being transported per a defined area and period of time. Measurements were performed according to the European Group for Efficacy Measurements on Cosmetics and Other Topical Products (EEMCO) guidance for the assessment of transepidermal water loss [15], the TEWL value is expressed in $\mathrm{g} / \mathrm{m}^{2} / \mathrm{h}$.

The measuring principle of the WCE is based on the capacitance method. The measurements were performed according to the EEMCO guidance for assessment of stratum corneum hydration [16]. The value considered is the mean of three measurements on adjacent sites of the forearm expressed as arbitrary units. As the measurement time is short, measurement errors caused by skin deformations or evaporation build-up can be excluded. 


\section{Statistical analysis}

All values are expressed as mean \pm SD. Transepidermal water loss and WCE values and other variables were normally distributed as assessed by the KolmogorovSmirnov test.

The parametric test, ANOVA for repeated measures, was applied for comparisons of the 3 paired means $( \pm$ SD) of TEWL and WCE (taken at DO, D30 and D60) of the two volar forearms (RF and LF) of each group (argan and olive). When the ANOVA test for repeated measures was significant, the Bonferroni test was applied to perform post hoc pairwise multiple comparisons within the three paired means $( \pm$ SD) of TEWL and WCE.

The Student's $t$-test for independent samples was used to compare the mean of the TEWL and WCE between the two forearms of each group as well as those of two forearms between the olive and argan group at the beginning of the study (at D0) to prove the homogeneity of the results and at the end of the study (at D60) to support the results of ANOVA for repeated measures.

Differences were considered to be statistically significant at $p<0.05$ and the statistical software SPSS version 10.0 was used.

\section{Results}

Baseline demographic and clinical characteristics of participants treated with argan and olive oil in both argan group and olive group are shown in Table I.

\section{Results of the untreated forearm or right volar forearm}

In the group consuming argan oil, the paired means $( \pm$ SD) of TEWL and WCE values in function of time (D0, D30 and D60) show a statistically significant difference between at least two means ( \pm SDs) of TEWL (Fig. 3A) and WCE (Fig. 4A) with a level of significance $p=0.023$ and $p=0.001$, respectively. This statistically significant difference exists between the D0 and the D60 mean $( \pm$ SD) for TEWL $(p=0.043)$ (Fig. 3A) and WCE ( $p=0.002)$ (Fig. 4A) and between the D30 and the D60 mean $( \pm$ SD) for TEWL $(p=0.044)$ (Fig. 3A) and WCE ( $p=0.019$ ) (Fig. 4A). While no statistically significant difference was observed between at least two means ( \pm SDs) of TEWL $(p>0.05)$ (Fig. 3B) and WCE values $(p>0.05)$ (Fig. 4B) in the group consuming olive oil. Between the group consuming argan oil and that consuming olive oil, there was no statistically significant difference in the unpaired means $( \pm$ SD) of TEWL $(p>0.05)$ and the WCE values $(p>0.05)$ at baseline (D0) but it became statistically significant after 60 days' period (D60) in favor of the group consuming argan oil for the TEWL $(p=0.046)$ and the WCE values $(p=0.047)$ (Table II).
Tab. I. Baseline demographic and clinical characteristics of argan and olive groups

\begin{tabular}{lcc}
\hline Item & $\begin{array}{c}\text { Olive group } \\
\text { (Control) }\end{array}$ & Argan group \\
\hline$N$ & 30 & 30 \\
\hline Age (years) & $55.13 \pm 6.45$ & $55.84 \pm 5.91$ \\
\hline Body weight $(\mathrm{kg})$ & $70.02 \pm 12.27$ & $69.94 \pm 11.85$ \\
\hline Height $(\mathrm{m})$ & $1.58 \pm 0.06$ & $1.57 \pm 0.05$ \\
\hline $\mathrm{TC}(\mathrm{g} / \mathrm{l})$ & $2.10 \pm 0.33$ & $2.13 \pm 0.35$ \\
\hline $\mathrm{LDL}-\mathrm{C}(\mathrm{g} / \mathrm{l})$ & $1.30 \pm 0.31$ & $1.34 \pm 0.31$ \\
\hline $\mathrm{HDL}-\mathrm{C}(\mathrm{g} / \mathrm{l})$ & $0.58 \pm 0.12$ & $0.58 \pm 0.15$ \\
\hline $\mathrm{TG}(\mathrm{g} / \mathrm{l})$ & $1.10 \pm 0.56$ & $1.09 \pm 0.51$ \\
\hline $\mathrm{All}$ ( $)$ & &
\end{tabular}

All values are presented as unadjusted mean \pm SD.

TC - total cholesterol, LDL-C - low-density lipoprotein cholesterol,

HDL-C - high-density lipoprotein cholesterol, TG - triglycerides

\section{Results of the treated forearm or left volar forearm}

In the group consuming argan oil, the paired means $( \pm$ SD) of TEWL and WCE values in function of time (D0, D30 and D60) show a statistically significant difference between at least two means ( \pm SDs) of TEWL and WCE with a level of significance $p=0.01$ (Fig. 3C) and $p<0.001$ (Fig. 4C), respectively, and with a level of significance $p=0.009$ (Fig. 3D) and $p<0.001$ (Fig. 4D), respectively, in the group consuming olive oil. This statistically significant difference exists between the DO and the D60 mean $( \pm$ SD) for TEWL $(p=0.012$ in the group consuming argan oil and $p=0.016$ in the group consuming olive oil) (Fig. 3C, D) and WCE $(p<0.001$ in the two groups) (Fig. 4C, D) and between the D30 and the D60 mean $( \pm$ SD) for TEWL $(p=0.009$ in the group consuming argan oil and $p=0.048$ in the group consuming olive oil) (Fig. 3C, D) and WCE ( $p=0.031$ in the group consuming argan oil and $p=0.046$ in the group consuming olive oil) (Fig. 4C, D). Between the group consuming argan oil and that consuming olive oil, there was no statistically significant difference in the unpaired means $( \pm$ SD) of TEWL $(p>0.05)$ and WCE values $(p>0.05)$ (Table II) at baseline (D0) and after 60 days' period (D60).

\section{Comparison of topical argan oil treated and untreated forearm or left volar forearm and right volar forearm in the two groups}

Between the group consuming argan oil and that consuming olive oil, there was no statistically significant difference in the unpaired means $( \pm$ SD) of TEWL $(p>0.05)$ and WCE values $(p>0.05)$ (Table III) at baseline (D0) but it became statistically significant after 60 days' period (D60) in favor of the LF in the group con- 

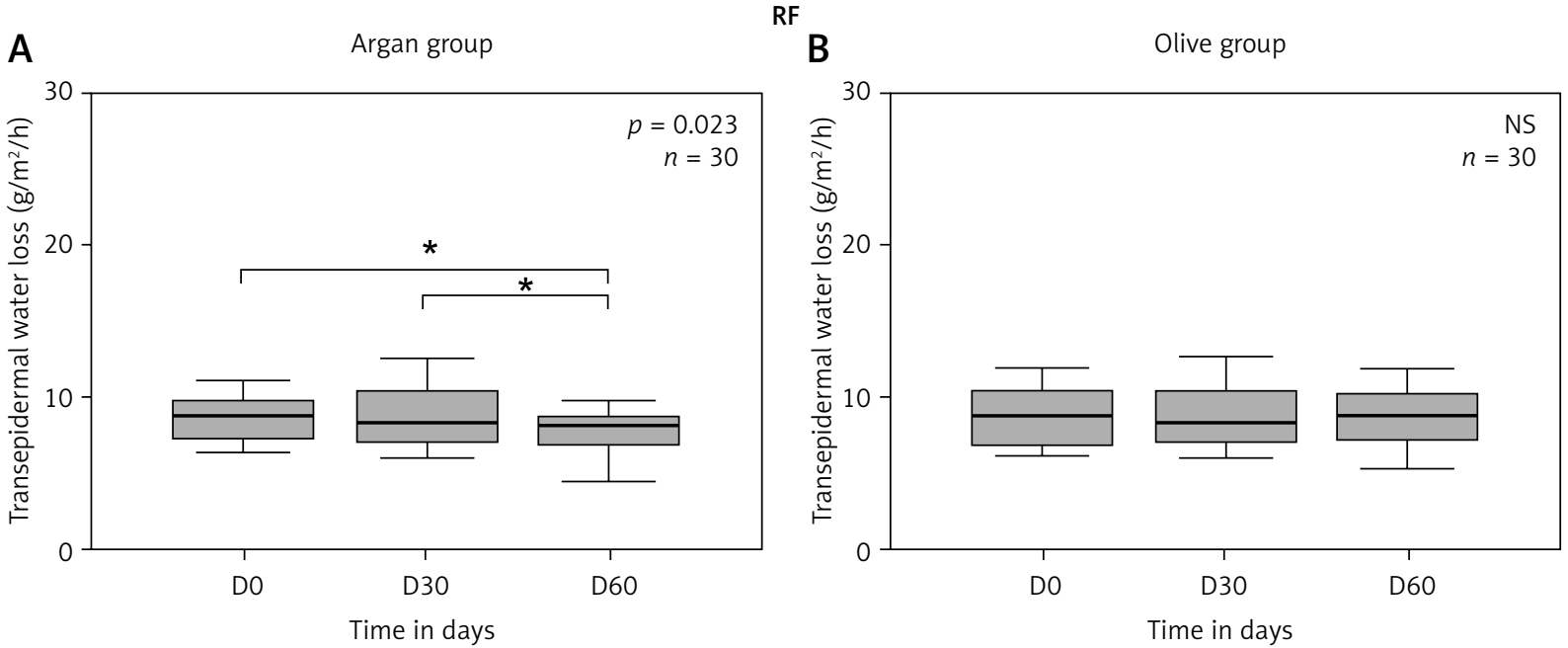

C

LF
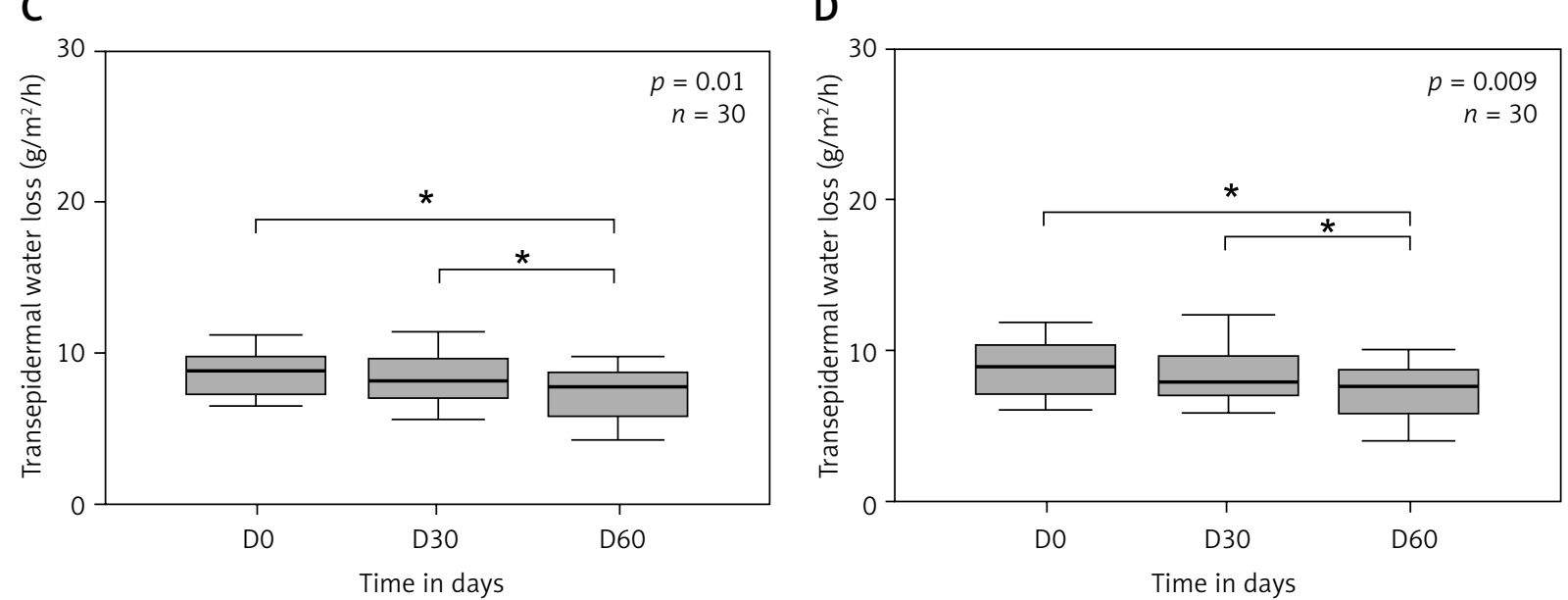

Fig. 3. Volar forearm transepidermal water loss (TEWL) evolution after oils consumption and/or argan oil application. A) At the right volar forearm (RF) in the group consuming argan oil. B) At the right volar forearm (RF) in the group consuming olive oil. C) At the left volar forearm (LF) in the group consuming argan oil. D) At the left volar forearm (LF) in the group consuming olive oil. The difference between the 3 paired means ( \pm SD) of TEWL (taken at D0, D30 and D60) of the two volar forearms (RF and LF) of each group (argan and olive) were analyzed using ANOVA test for repeated measures followed by Bonferroni post-hoc test (if ANOVA test for repeated measures was statistically significant). $p<0.05$ was considered statistically significant (results of ANOVA test for repeated measures). NS: ANOVA test for repeated measures shows no statistically significant difference $(p>0.05) .{ }^{*} p<0.05$ was considered statistically significant (results of Bonferroni post-hoc test)

suming olive oil with $p=0.023$ and $p=0.012$ for the TEWL and the WCE (Table III) values, respectively.

\section{Discussion}

This study has shown that the daily consumption and application of argan oil during a 60 days' period have a beneficial effect on skin hydration by restoring the barrier function and maintaining water-holding capacity of the skin in postmenopausal women.

In postmenopausal women, the decrease in endogenous estrogen level negatively affects the epidermal barrier function, promoting the occurrence of dry skin, which agrees with the study of Dunn et al. [6]. The improvement of skin hydration after administration of es- trogen in the form of HRT [6] can be linked to the role of estrogen in stimulation of epidermal keratinocyte proliferation [17] involved in the epidermal lipids synthesis, which favors the maintenance of the epidermal barrier function.

The barrier function of the epidermis was evaluated most often by measuring the TEWL, a means used for detecting the disruption of the barrier function [18], because the values of TEWL are very high in the event of disruption of the barrier function of the epidermis [6], observed in the case of menopause and skin diseases.

In our study, measurements of TEWL were performed to evaluate the influence of argan oil treatment on the barrier function of the skin. As well, the measures of WCE are used to confirm the results of TEWL since the 
A

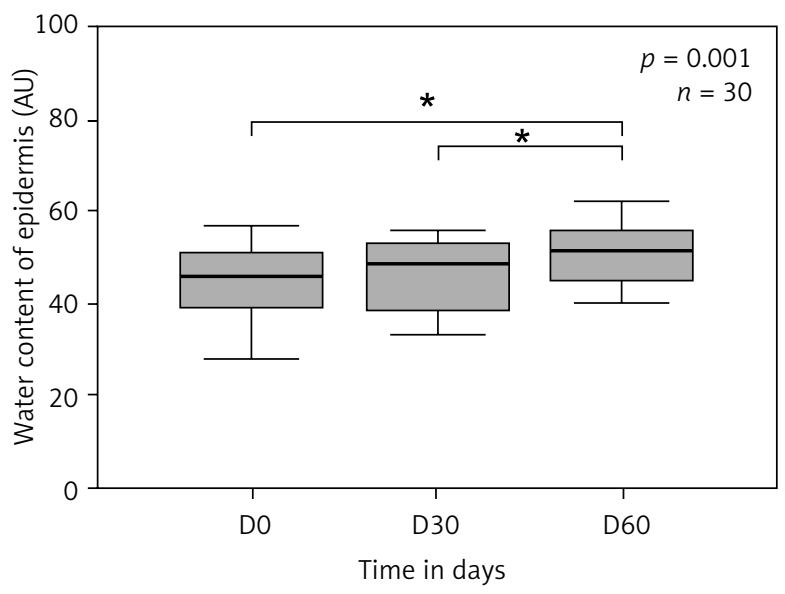

C

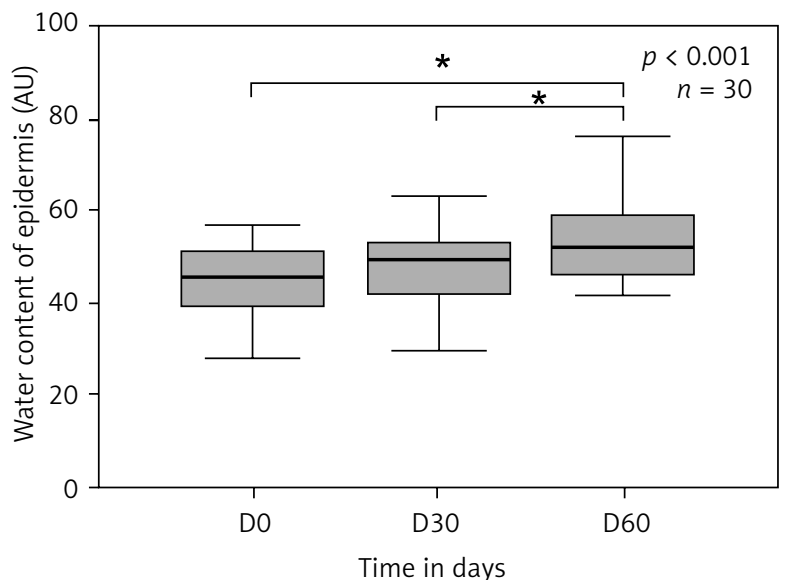

B

Olive group

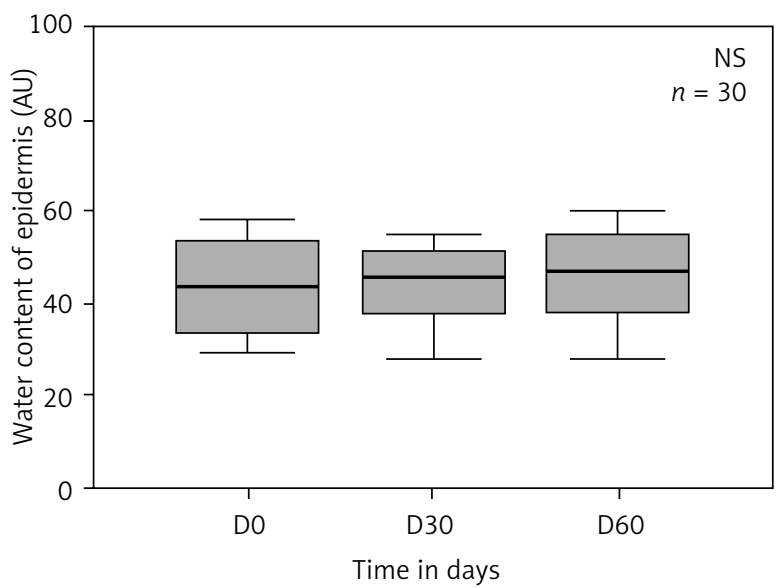

LF

D

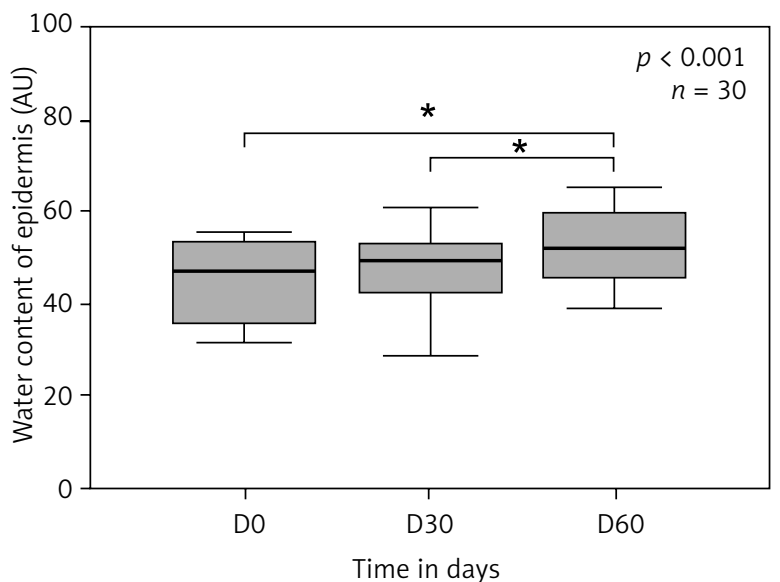

Fig. 4. Volar forearm water content of epidermis (WCE) evolution after oils consumption and/or argan oil application. A) At the right volar forearm (RF) in the group consuming argan oil, B) at the right volar forearm (RF) in the group consuming olive oil, C) at the left volar forearm (LF) in the group consuming argan oil, D) at the left volar forearm (LF) in the group consuming olive oil. The difference between the 3 paired means ( \pm SD) of WCE (taken at D0, D30 and D60) of the two volar forearms (RF and LF) of each group (argan and olive) were analyzed using ANOVA test for repeated measures followed by Bonferroni post-hoc test (if ANOVA test for repeated measures was statistically significant). $p<0.05$ was considered statistically significant (results of ANOVA test for repeated measures). NS - ANOVA test for repeated measures shows no statistically significant difference $(p>0.05) .{ }^{*} p<0.05$ was considered statistically significant (results of Bonferroni post-hoc test)

Tab. II. The intergroup comparison of transepidermal water loss (TEWL) and water content of epidermis (WCE) volar forearm between the two groups before (D0) and after sixty days (D60) of oils consumption and/or argan oil application

\begin{tabular}{lllccc}
\hline & & & Olive group $(n=30)$ & Argan group $(n=30)$ & $p$ \\
\hline \multirow{2}{*}{ TEWL $\left(\mathrm{g} / \mathrm{m}^{2} / \mathrm{h}\right)$} & $\mathrm{RF}$ & D0 & $8.93 \pm 2.2$ & $8.92 \pm 2.44$ & $>0.05$ \\
\cline { 2 - 5 } & & D60 & $8.57 \pm 1.99$ & $7.65 \pm 1.48$ & 0.046 \\
\cline { 2 - 5 } & \multirow{2}{*}{ LF } & $8.93 \pm 2.2$ & $8.92 \pm 2.44$ & $>0.05$ \\
\cline { 2 - 5 } & & D0 & $7.44 \pm 1.75$ & $7.32 \pm 1.47$ & $>0.05$ \\
\hline \multirow{2}{*}{ DCE (AU) } & RF & D0 & $44.38 \pm 9.99$ & $44.74 \pm 7.68$ & $>0.05$ \\
\cline { 2 - 5 } & & D60 & $46.79 \pm 9.22$ & $50.97 \pm 6.53$ & 0.047 \\
\cline { 2 - 5 } & LF & D0 & $44.79 \pm 8.49$ & $44.74 \pm 7.68$ & $>0.05$ \\
\cline { 2 - 5 } & & D60 & $52.62 \pm 8.12$ & $54.59 \pm 10.74$ & $>0.05$ \\
\hline
\end{tabular}

All values are presented as mean \pm SD.

$\mathrm{AU}$ - arbitrary units, RF - right volar forearm, LF - left volar forearm.

Mean group comparisons were conducted using Student's $t$-test for independent samples.

$p<0.05$ was considered statistically significant. 
Tab. III. The intergroup comparison of transepidermal water loss (TEWL) and water content of epidermis (WCE) between the two volar forearms (right [RF] and left [LF]) before (D0) and after sixty days (D60) of oils consumption and/or argan oil application in the two groups

\begin{tabular}{|c|c|c|c|c|c|}
\hline & & & RF & LF & $p$ \\
\hline \multirow[t]{4}{*}{ TEWL $\left(\mathrm{g} / \mathrm{m}^{2} / \mathrm{h}\right)$} & \multirow{2}{*}{$\begin{array}{l}\text { Argan group } \\
(n=30)\end{array}$} & D0 & $8.92 \pm 2.44$ & $8.92 \pm 2.44$ & $>0.05$ \\
\hline & & D60 & $7.65 \pm 1.48$ & $7.32 \pm 1.47$ & $>0.05$ \\
\hline & \multirow{2}{*}{$\begin{array}{l}\text { Olive group } \\
(n=30)\end{array}$} & D0 & $8.93 \pm 2.20$ & $8.93 \pm 2.2$ & $>0.05$ \\
\hline & & D60 & $8.57 \pm 1.99$ & $7.44 \pm 1.75$ & 0.012 \\
\hline \multirow[t]{4}{*}{ WCE (AU) } & \multirow{2}{*}{$\begin{array}{l}\text { Argan group } \\
(n=30)\end{array}$} & DO & $44.74 \pm 7.68$ & $44.74 \pm 7.68$ & $>0.05$ \\
\hline & & D60 & $50.97 \pm 6.53$ & $54.59 \pm 10.74$ & $>0.05$ \\
\hline & \multirow{2}{*}{$\begin{array}{l}\text { Olive group } \\
(n=30)\end{array}$} & DO & $44.38 \pm 9.99$ & $44.79 \pm 8.49$ & $>0.05$ \\
\hline & & D60 & $46.79 \pm 9.22$ & $52.62 \pm 8.12$ & 0.023 \\
\hline
\end{tabular}

All values are presented as mean $\pm S D$

$\mathrm{AU}$ - arbitrary units, RF - right volar forearm, LF - left volar forearm.

Mean of RF and LF comparisons were conducted using Student's $t$-test for independent samples.

$p<0.05$ was considered statistically significant.

Tab. IV. Composition of argan and olive oil [21-23]

\begin{tabular}{|c|c|c|}
\hline & Argan oil & Olive oil \\
\hline \multicolumn{3}{|l|}{ Fatty acids (\%) } \\
\hline Palmitic acid & 13.4 & 10.4 \\
\hline Stearic acid & 5.1 & 2.76 \\
\hline Oleic acid & 44.8 & 71.0 \\
\hline Linoleic acid & $31-35$ & $4.5-16.1$ \\
\hline Linolenic acid & 0.1 & 1.04 \\
\hline Squalene (mg/100 g) & 313 & 499 \\
\hline \multicolumn{3}{|l|}{ Sterols (mg/100 g oil) } \\
\hline Schottenol & 142 & 0 \\
\hline Spinasterol & 115 & 0 \\
\hline Stigmasta-8.22-dien-3 $\beta$-ol & 9 & 0 \\
\hline$\beta$-Sitosterol & 0 & 156 \\
\hline Campesterol & 0 & 12 \\
\hline Others & 29 & 151 \\
\hline Total & 295 & 319 \\
\hline \multicolumn{3}{|l|}{ Tocopherols (mg/kg oil) } \\
\hline $\mathrm{A}$ & 35 & 190 \\
\hline$\Delta$ & 122 & 42 \\
\hline$\Gamma$ & 480 & 26 \\
\hline Total & 636 & 257 \\
\hline \multicolumn{3}{|l|}{ Phenolic compounds ( $\mu \mathrm{g} / \mathrm{kg}$ oil) } \\
\hline Vanillic acid & 67 & 359 \\
\hline Syringic acid & 37 & 0 \\
\hline Ferulic acid & 3147 & 51 \\
\hline Tyrosol & 12 & 19573 \\
\hline Others & 0 & 773000 \\
\hline Total & 3263 & 792983 \\
\hline
\end{tabular}

decrease in TEWL induces the increase in WCE, promoting the maintenance of skin water-holding capacity [19].

The results of the RF comparison show an improvement in skin hydration after 60 days of alimentary argan oil consumption, which was characterized by a decrease in TEWL and an increase in WCE; both being statistically significant. While no statistically significant improvement in skin hydration has not been provided after the consumption of olive oil. As well, the comparison of the RF between the two groups at D60 showed a statistically significant improvement in skin hydration in favor of the group consuming argan oil after 60 days of consumption. Transepidermal water loss measurements are sensitive to climatic variations due to the fact that when the relative humidity of the surrounding air is $100 \%$, the TEWL decreases to almost $0 \mathrm{~g} / \mathrm{m}^{2} / \mathrm{h}$ and inversely [20]. So our results would not be due to changes in TEWL values linked to climatic fluctuations, since the measurements of TEWL were taken in climatic controlled conditions (temperature of $22 \pm 1^{\circ} \mathrm{C}$, relative humidity of $56 \pm 1 \%$ ), and WCE values have been also changed in parallel to TEWL changes. But these changes of TEWL may be due to the content of argan oil in linoleic acid (Table IV), higher than that observed in the olive oil (Table IV), which agrees with the study of Cosgrove et al. in which the higher dietary intake of linoleic acid has a beneficial role in reducing the chances of developing senile dryness in middle-aged women [24]. It is also likely that this difference is due to a synergy between particular components of unsaponifiable fractions (Table IV) actually quite different between the two oils, and their levels of linoleic acid.

In both groups, the results of the LF comparison show a statistically significant improvement in skin hydration after 60 days of oils treatment. In the group consuming argan oil, these results of the LF reflect a more 
synergetic effect of the consumption and application of argan oil. While, in the group consuming olive oil, the results of the LF will be due only to the effect of argan oil application, since the consumption of olive oil has made no statistically significant improvement. This effect of argan oil application would not be due to the occlusive effect of argan oil, because the measurements were not taken immediately after the last application, as well as no significant change in TEWL and WCE values was seen after 30 days of topical application.

These results would be also due to the high content of linoleic acid in argan oil (Table IV), because linoleic acid enters in the composition of the ceramides of epidermal cement and principally that of ceramide- 1 subclass. As ceramide-1 plays a key role in the organization of the stratum corneum lipids [25], the replacement of ceramide-1 linoleate with oleate in the case of essential fatty acid deficiency induced by the dramatic perturbations in the ultrastructure of the stratum corneum lipids [26], which can be restored after topical application of essential fatty acid supported by the study of Imokawa et al. [27], after 2 weeks' application of pseudo-esterlinked acyl ceramide with linoleic acid (BRS-001).

This beneficial effect of linoleic acid, on improvement of skin hydration is achieved by the activation of peroxisome proliferator-activated hormone which provides the activation of epidermal keratinocyte differentiation [28], leading to the synthesis of the cement epidermal lipids for ensuring the restoration of the barrier function [29] disrupted during menopause characterized by the decrease in the TEWL [27], which agrees with our results. Once again, we cannot exclude the likely involvement of a synergy between the specific components within each oil (Table IV).

The limitation of our study was the short duration of the intervention (60 days), the extension of this period for one month or more was not possible, since most of the volunteers had other commitments. In addition to the inclusion of linoleic acid and other argan oil chemical components (vitamin E, phenols, phenolic acid, and other compounds) in olive oil with the exception of two essential sterols (schottenol and spinasterol) and syringic acid (Table IV) that have no known effects on skin hydration, the lack of compliance assessment, and the lack of isolation for the active ingredient(s) in argan oil.

The advantage of this study is that we have shown the moisturizing effect of alimentary and cosmetic argan oil. As well, no side effects have been noted with argan oil treatments during the study period. In addition to emollient properties of this oil in improving skin hydration proven through this study, our population has also benefited from the positive effect of argan oil in prevention of disruptions or diseases related to menopause [11, 22]. Parts of them (lipid and hormone profiles, antioxidant effect) are simultaneously investi- gated by the other members of the consortium of the research team.

\section{Conclusions}

The consumption and application of alimentary and cosmetic argan oil, respectively, showed an ameliorative effect on skin hydration of postmenopausal women after a 60 days' period. This moisturizing effect even internally, makes argan oil a separate moisturizer.

\section{Acknowledgments}

We are indebted to "Hassan II Academy of Science" that funded our study, to "Ibn Al Baytar association" that was involved in the management of this study, to all women who participated in this study and also to Miss Rouchdi Mounia and Miss Zniber Meryem who accompanied these women during the intervention period.

\section{Disclosure}

Hassan II Academy of Science financially supported this study.

Authors report no conflicts of interest.

\section{References}

1. Nakagawa N, Sakai S, Matsumoto M, et al. Relationship between NMF (lactate and potassium) content and the physical properties of the stratum corneum in healthy subjects. J Invest Dermatol 2004; 122: 755-763.

2. Ponec M, Weerheim A, Lankhorst P, Wertz P. New acylceramide in native and reconstructed epidermis. J Invest Dermatol 2003; 120: 581-588.

3. Jungersted JM, Hellgren LI, Jemec GBE, Agner T. Lipids and skin barrier function - a clinical perspective. Contact Dermatitis 2008; 58: 255-262.

4. Raney SG, Hope MJ. The effect of bilayer and hexagonal HII phase lipid films on transepidermal water loss. Exp Dermatol 2006; 15: 493-500.

5. Breternitz M, Kowatzki, D, Langenauer M, et al. Placebo-controlled, double-blind, randomized, prospective study of a glycerol-based emollient on eczematous skin in atopic dermatitis: biophysical and clinical evaluation. Skin Pharmacol Physiol 2008; 21: 39-45.

6. Dunn L, Damesyn M, Moore A, et al. Does estrogen prevent skin aging?: results from the First National Health and Nutritional Examination Survey. Arch Dermatol 1997; 133: 339-342.

7. Antoine C, Liebens F, Carly B, et al. Influence of HRT on prognostic factors for breast cancer: a systematic review after the Women's Health Initiative trial. Hum Reprod 2004; 19: 741-756.

8. Berrougui H, Ettaib A, Herrera Gonzalez MD, et al. Hypolipidemic and hypocholesterolemic effect of argan oil (Argania spinosa L.) in Meriones shawi rats. J Ethnopharmacol 2003; 89: 15-18.

9. Cherki M, Berrougui $H$, Drissi A, et al. Argan oil: Which benefits on cardiovascular diseases? Pharmacol Res 2006; 54: 1-5.

10. Bellahcen S, Mekhfi H, Ziyyat A, et al. Prevention of chemically induced diabetes mellitus in experimental animals by virgin argan oil. Phytother Res 2012; 26: 180-185.

11. Azizi F, Ainy E. Coronary heart disease risk factors and menopause: a study in 1980 Tehranian women, the Tehran Lipid and Glucose Study. Climactric 2003; 6: 330-336.

12. Bellakhdar J. La pharmacopée marocaine traditionnelle. Ibis Press, Paris 1997; 486-488. 
13. Guillaume D, Charrouf Z. Argan oil and other argan products: use in dermocosmetology. Eur J Lipid Sci Technol 2011; 113: 403-408.

14. Derouiche A, Cherki M, Drissi A, et al. Nutritional intervention study with argan oil in man: effects on lipids and apolipoproteins. Ann Nutr Metab 2005; 49: 196-201.

15. Rogiers V. EEMCO guidance for the assessment of transepidermal water loss in cosmetic sciences. Skin Pharmacol Appl Skin Physiol 2001; 14: 117-128.

16. Berardesca E. EEMCO guidance for the assessment of stratum corneum hydration: Electrical methods. Skin Res Technol 1997; 3: 126-132.

17. Urano R, Sakabe K, Seiki K, Ohkido M. Female sex hormone stimulates cultured human keratinocyte proliferation and its RNA- and proteinsynthetic activities. J Dermatol Sci 1995; 9: 176-184.

18. Fluhr JW, Feingold KR, Elias PM. Transepidermal water loss reflects permeability barrier status: validation in human and rodent in vivo and ex vivo models. Exp Dermatol 2006; 15: 483-492.

19. Fluhr JW, Miteva M, Primavera G, et al. Functional assessment of a skin care system in patients on chemotherapy. Skin Pharmacol Physiol 2007; 20: 253-259.

20. Pirot F, Falson F. Skin barrier function. In: Agache P, Humbert P (eds.). Measuring the skin. Springer-Verlag, Berlin 2004.

21. Charrouf Z, Guillaume D. Argan oil: Occurrence, composition and impact on human health. Eur J Lipid Sci Technol 2008; 110: 632-636.

22. Ollivier D, Artaud J, Pinatel C, et al. Triacylglycerol and fatty acid compositions of French virgin olive oils. Characterization by chemometrics. J Agric Food Chem 2003; 51: 5723-5731.
23. Khallouki F, Younos C, Soulimani R, et al. Consumption of argan oil (Morocco) with its unique profile of fatty acids, tocopherols, squalene, sterols and phenolic compounds should confer valuable cancer chemopreventive effects. Eur J Cancer Prev 2003; 12: 67-75.

24. Cosgrove MC, Franco OH, Granger SP, et al. Dietary nutrient intakes and skin-aging appearance among middle-aged American women. Am J Clin Nutr 2007; 86: 1225-1231.

25. Bouwstra JA, Gooris GS, Dubbelaar FER, et al. Role of ceramide 1 in the molecular organization of the stratum corneum lipids. J Lipid Res 1998; 39: 186-196.

26. Hou SYE, Mitra AK, White SH, et al. Membrane structures in normal and essential fatty acid-deficient stratum corneum: characterization by ruthenium tetroxide staining and X-ray diffraction. J Invest Dermatol 1991; 96: 215-223.

27. Imokawa G, Yada Y, Higuchi K, et al. Pseudo acylceramide with linoleic acid produces selective recovery of diminished cutaneous barrier function in essential fatty acid deficient rats and has an inhibitory effect on epidermal hyperplasia. J Clin Invest 1994; 94: 89-96.

28. Sertznig P, Seifert M, Tilgen W, Reichrath J. Peroxisome proliferatoractivated receptors (PPARs) and the human skin: importance of PPARs in skin physiology and dermatologic diseases. Am J Clin Dermatol 2008; 9: 15-31.

29. Man MQ, Choi EH, Schmuth M, et al. Basis for improved permeability barrier homeostasis induced by PPAR and LXR activators: liposensors stimulate lipid synthesis, lamellar body secretion, and post-secretory lipid processing. J Invest Dermatol 2006; 126: 386-392. 